\title{
The Modulation of the Retinal Relay to the Cortex in the Dorsal Lateral Geniculate Nucleus
}

\author{
A M SILLITO and P C MURPHY \\ London
}

\section{Summary}

The translation of the retinal input through the dorsal lateral geniculate nucleus (dLGN) to the visual cortex is highly dependent on a range of influences. This article reviews the available evidence. One of the influences, the corticofugal projection to the dLGN from layer VI of the visual cortex, provides a synaptic input which in magnitude exceeds that from the retina. This makes direct synaptic contact on relay cells and the intrinsic and perigeniculate inhibitory interneurones influencing their activity. The corticofugal system appears to be spatially organised in such a way that for any given region in the dLGN, there is a central zone comprising an overlying field with facilitatory effect, and a surrounding zone with inhibitory influence. The extent to which these overlap is open to question at present. The inhibitory effect of the corticofugal projection can be clearly seen in its contribution to the length tuning of dLGN cells when tested with drifting bars. On average dLGN cells exhibit a very high degree of length tuning, matching that of cortical hypercomplex cells. Removal of the corticof ugal influence causes a radical reduction in this, shifting the mean reduction in peak response with increasing bar length from $71 \%$ to $43 \%$. One consequence of this corticof ugal effect is that the selectivity of the dLGN cell receptive field towards stimuli spatially restricted to the vicinity of the centre mechanism, is as good for moving bars as it is for stationary flashing spots. The retinal output to dLGN relay cells appears to be mediated by excitatory amino acid receptors, of both NMDA and non-NMDA categories. The non-NMDA receptors appear to provide an initial level of depolarisation which enables the operation of the voltage dependent NMDA receptor channels. The NMDA receptor however sits as a critical gate regulating the transmission of retinal information in the dLGN, when it is blocked visual responses are virtually eliminated. Its voltage dependency makes it crucially dependent on the complex pattern of excitatory and inhibitory influences from the cortex and the "non-specific" modulatory influence of the cholinergic system.

The output from the retina reaches the visual cortex via relay cells in the dorsal lateral geniculate nucleus (dLGN). Whilst it is common to depict this as involving a more or less direct translation of the properties of retinal ganglion cells to dLGN cells, it is becoming increasingly apparent that the retina's access to this pathway is conditional on many factors. These include neuromodulatory inputs from the brainstem, inhibitory control mediated by local inhibitory interneurones, and a massive projection back from the visual cortex. The corticofugal projection, in particular, is substantially larger than that from the retina, and there are good grounds for believing that its synaptic connections have the same potential effectiveness. The implication here is that the cortex must exert a potent control over the information that it receives from the eye. In this article we shall consider, from two viewpoints, 


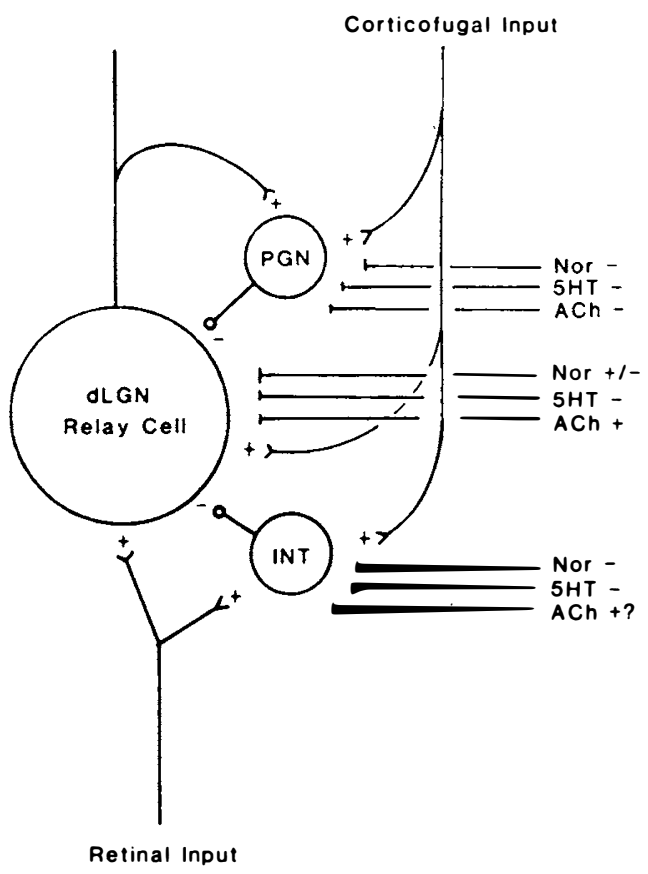

Fig. 1. Diagram summarising the main connections influencing a $d L G N$ relay cell. These include two groups of inhibitory interneurones, the intrinsic interneurones (INT) and perigeniculate cells (PGN), together with the cholinergic (ACh), noradrenergic (Nor) and serotinergic (5-HT) extrinsic modulatory inputs. Note that corticofugal projection makes direct excitatory contacts on relay cells and both sorts of inhibitory interneurone.

the ways in which the corticofugal projection may enact this control. One concerns the direct observations available to date regarding the modulation of visual response properties by corticofugal fibres. The other refers to the nature of the neurotransmitter receptors that are thought to mediate retinal output, and the properties of one of these that may make dLGN responses critically vulnerable to the influence of the corticofugal system.

\section{Basic synaptic organisation of the dLGN}

A simplified summary of the types of connection seen in the dLGN is given in Figure 1. The retinal afferents synapse on the proximal dendrites of relay cells, while the descending corticofugal excitatory input arrives on the more distal portions. ${ }^{1}$ These cells also receive two types of inhibitory input, one from intrinsic inhibitory interneurones within the dLGN and the other from cells in the overlying perigeniculate nucleus (PGN). Corticofugal fibres make excitatory contacts on both types of inhibitory neurone. It is likely that the axons of the intrinsic inhibitory interneurones make conventional synaptic contacts on the shafts of relay cell dendrites. In addition, however, they take part in "triadic synapses". Here the dendrites of the interneurone are presynaptic to a dendritic spine of the relay cell, but together they are postsynaptic to the retinal afferent terminals. $^{2}$ This creates the basis for a very specific control of the transfer of information from the retinal afferent to the target cell. Triadic synapses are associated with dendritic spines rather than the shafts of relay cell dendrites. " $X$ " cells seem to be much more prominently spined than "Y" cells, and the influence of this type of interaction is therefore presumed to be reflected primarily in the properties of dLGN " $X$ "cells. ${ }^{1,3}$ The cells in the perigeniculate nucleus are driven by relay cell collaterals, and mediate an inhibitory feedback to both relay cells and intrinsic inhibitory interneurones. ${ }^{4}$ All of these interactions are modulated by at least three extrinsic brain stem inputs, a serotinergic input from the dorsal raphe nuclei, a cholinergic input from the central tegmental field and a noradrenergic input from the locus coeruleus. ${ }^{5,6,7}$ From the viewpoint of the present discussion the most important points to note are the magnitude of the corticofugal projection relative to the retinal input, and the distribution of the corticofugal fibres to both the relay cells and the two groups of inhibitory interneurones.

\section{Influence of the corticofugal system on visual processing}

Despite the magnitude of the corticofugal projection, and a number of previous studies of its possible function, there has until recently been little direct evidence to support any specific pattern of influence on visual responses. Several studies have examined the effects of cooling areas 17,18 and 19 on the responses of dLGN cells to a range of visual stimuli, including flashing spots and annuli. In the cat visual system, cooling the visual cortex has been reported both to increase and decrease the responses of dLGN cells to visual stimulation of receptive field centre or surround, and to affect the centre-surround balance. ${ }^{8,9}$ Whilst these 
studies make it clear that the corticofugal system exerts an effect that can be basically excitatory or inhibitory on the visual responses of a significant proportion of dLGN cells $(50-86 \%)$, there is no coherent logic to the type of effect observed. Possibly this is not altogether surprising, insofar as the stimuli used were those that might be regarded as effective for studying dLGN cells, but not those that are suitable for activating layer VI corticogeniculate cells. Visual cortical cells in the cat are best driven by moving bar stimuli of the appropriate orientation ${ }^{10}$ and are poorly activated by stationary flashing spots or annuli. The layer VI corticogeniculate cells in particular have been reported to show substantial length summation in the plane of their optimal orientation $^{11}$ and are likely to be maximally activated by a bar $4^{\circ}$ or more long, drifting over their receptive field. The effects of cortical cooling on the responses of dLGN cells to flashing spots and annuli are therefore likely to reflect the loss of the minimal level of synaptic input that may follow from the spontaneous activity of corticofugal cells (generally accepted to be low eg Gilbert ${ }^{11}$ ) or the residual responses elicited by the flashing stimuli themselves. In either case these effects would be at the lowest extreme of the spectrum of anticipated corticofugal influence, and could not be expected to reflect that which would be exerted when the corticofugal cells are appropriately engaged by an optimal stimulus configuration.

A much more effective approach was taken by McClurkin and Marrocco ${ }^{12}$ in the primate. They demonstrated that rotation of a radial grating, with a central aperture located over a dLGN cell receptive field, produced a corticofugally driven modulation of the response to a visual stimulus presented within the aperture. The effect was seen in approximately half the cells studied, and was blocked by cooling the cortex. They observed changes in both the spatial and temporal properties of dLGN cells, and were able to relate the alterations in spatial properties to shifts in the centre-surround balance. In a further paper $^{13}$ they attempted to examine the spatial structure of their corticofugal modulation, and concluded that individual dLGN cells received facilitatory and inhibitory influences driven by areas of cortex representing different regions of visual space. Broadly these could be described as a central inhibitory or facilitatory zone surrounded by an area of opposite effect. The methods of Marrocco and McClurkin did not allow them to distinguish the precise extent of the different components of this spatial organisation. However, their results are directly comparable with those obtained in the cat visual system by Tsumoto, Creutzfeldt and Legendy, ${ }^{14}$ who were able to examine this question in more detail. The study of Tsumoto et al involved different methods. They used iontophoretic glutamate application to excite cortical cells whilst recording the effect of this on single unit activity in the dLGN. They observed that where the receptive field location in the cortex and the dLGN were within $2^{\circ}$ of each other, excitatory effects could be seen, while beyond this inhibitory effects predominated up to a maximal separation of $3^{\circ}$. Close examination of their records suggests that inhibitory effects could be observed virtually throughout the region of "visual space" driving the corticofugal influence, but excitatory effects were restricted to a more central zone. This "centre-surround" pattern is reminiscent of the basic organisation of the geniculate and retinal ganglion cell fields.

In a recent series of experiments carried out in our own laboratory, we adopted a rather different approach to examining the role of the corticofugal system. This is based on several premises which need to be considered before discussing the main findings. Firstly, we have taken the view that the best way to demonstrate the action of the corticofugal system is to examine the responses of dLGN cells to a stimulus that itself drives cortical cells, and particularly layer VI cells. Secondly, we have made the assumption that varying the parameters of the stimulus in a way that changes the response of the cortical cells will also affect the magnitude of the corticofugal influence, and produce a corresponding perturbation in the response of the dLGN cells to that stimulus. This would be in addition to any direct impact of this procedure on dLGN cell responses, which would be seen in the absence of corticofugal feedback. Two aspects of the known response properties of the constituent cells in the system provided us with what seemed to be a direct access to this problem. It has become increasingly apparent that, when tested with moving bars, the cells in the dLGN are strongly length tuned; as much or 
more so than many cells that in the cortex would be 'classified as hypercomplex. ${ }^{15,16}$ This preference for short bars contrasts with the feature that seems to typify many of the corticofugal cells in layer VI, namely their elongated fields and preference for longer stimuli. At first sight this seems to present something of a paradox, since the long fields of the corticofugal cells can be regarded as presynaptic to the short dLGN fields. However, as well as their direct excitatory contacts on relay cells, corticofugal cells provide strong synaptic input to the two types of inhibitory interneurone that influence relay cell activity. It thus seems certain that any given relay cell will receive both inhibitory and excitatory inputs. Both might be expected to increase in magnitude as stimulus length increases, but the overall effect at any one length will depend upon the precise balance of the individual influences. This in turn will depend on details of the synaptic organisation at the level of the dLGN that are not as yet known, but from the work of Tsumoto et al ${ }^{14}$ one might presume that facilitatory effects could occur for

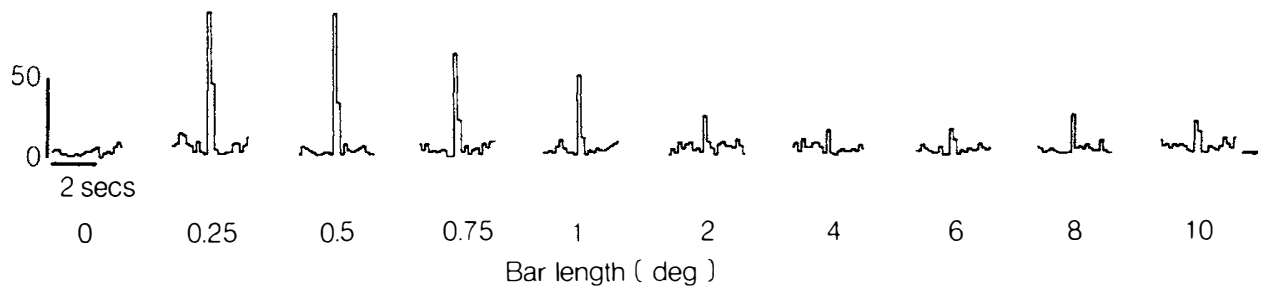

Fig. 2. Responses of an on-centre $Y$ cell in the dLGN to the motion of a bar of light of varying length over its receptive field. Peristimulus time histograms (PSTHs) are plotted with 150 msec bins and averaged over five trials. Vertical calibration shows range for 0-50 counts/150 msec bin.

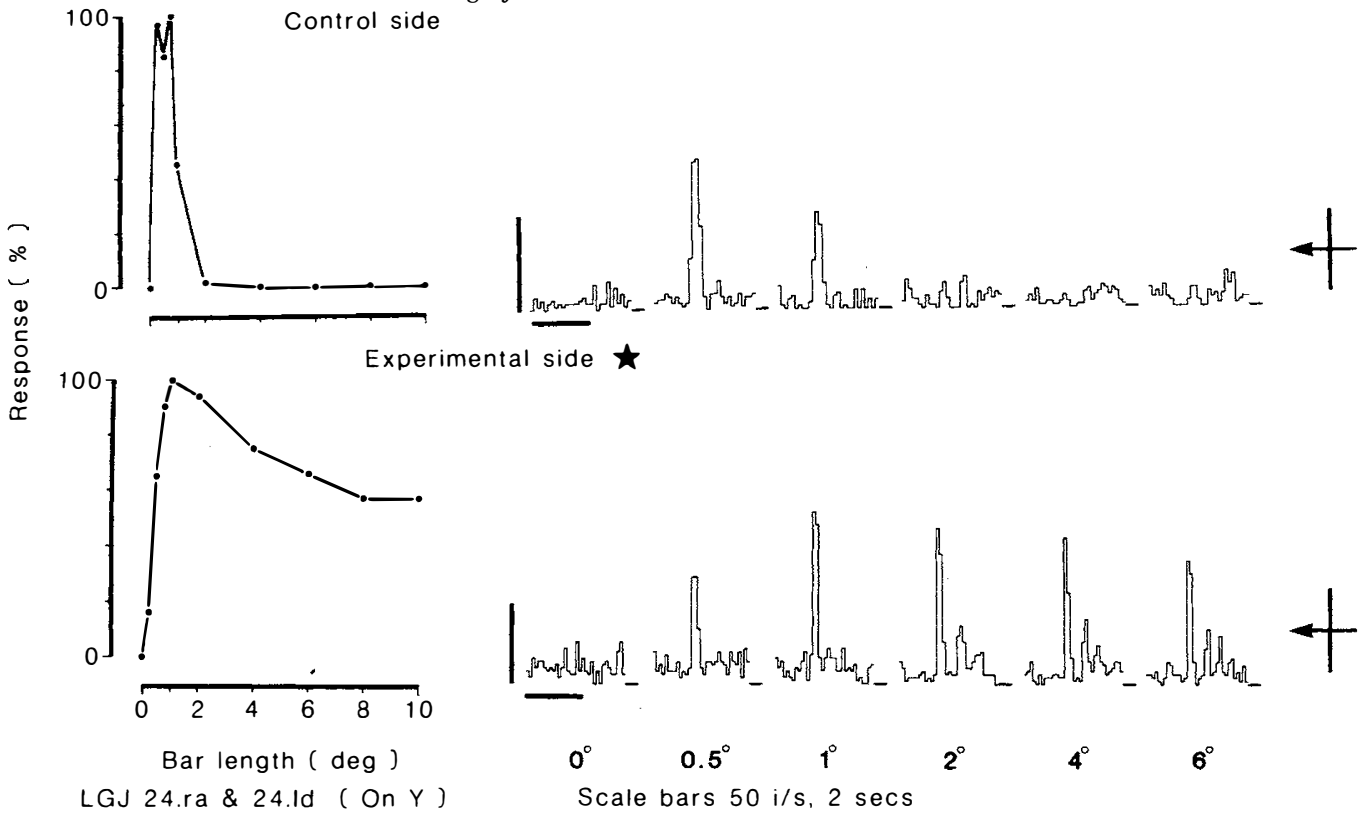

Fig. 3. Effect of corticofugal feedback on the length tuning of $d L G N$ cells. Records show the length tuning curves and individual PSTHs for the response of two on-centre Y cells to a bar of light of varying length moving over their receptive field. The length tuning curves plot the responses expressed as a percentage of the maximum seen to an optimal length stimulus. The upper records show a cell with corticofugal feedback, the lower records that of cell recorded in the opposite dLGN in the same preparation, but without corticofugal feedback. Vertical calibration bars on PSTHs refer to range for 0-50 counts/bin, horizontal two secs. Bin size was 100 msecs, contrast was 150 on $50 \mathrm{~cd} \mathrm{~m}^{-2}$ and bar width $0.5^{\circ}$. 
shorter bar lengths and inhibitory effects predominate as bar length goes up and encroaches on the "surround" of the "corticofugal field". These ideas led us to speculate that a predominantly inhibitory influence of corticofugal origin could contribute to the strong length tuning seen in many dLGN cells.

\section{The corticofugal contribution to dLGN cell} length tuning

Taken as a whole, the normal population of dLGN cells . with good centre-surround antagonism show a high level of length tuning. The records in Figure 2 show the responses of one such cell, an on-centre $Y$ cell, in response to a bar of light of varying length moving over its receptive field. The radical fall-off in response at bar lengths over $0.75^{\circ}$ is immediately apparent. One can quantify the degree of length tuning in terms of the reduction in response seen at longer bar lengths as compared with that at the optimal length. For a population of $100 \mathrm{dLGN}$ cells sampled in preparations with corticofugal feedback, the mean reduction between the peak value and the average response seen over the plateau of the tuning curve, was $77 \%(+/-2 \%$ s.e.m. $){ }^{16}$ How is this property affected by the corticofugal system? We explored this issue by simultaneously recording from cells in the left and right dLGN, and then removing areas 17 and 18 of the visual cortex on one side. We compared the dLGN cell response properties on the two sides before and after this procedure, with particular reference to length tuning and centresurround antagonism. ${ }^{17}$ An example of our data is given in Figure 3 and summarised in the histograms in Figure 4. The central point is that there is a marked difference in the length tuning of cells with and without corticofugal feedback. In its absence, length tuning is greatly reduced, with the mean shifting from an average of $71 \%$ on the control side to $43 \%$ on the side without feedback. The histograms in Figure 4 show the distribution of length tuning for the two conditions. The neurones were categorised into 10 groups based on the magnitude of the response reduction seen at increasing lengths, where those in group 10 showed maximal (90-100\%) reduction in response and those in group 1 minimal reduction. It is generally accepted in the
DISTRIBUTION OF END-INHIBITION

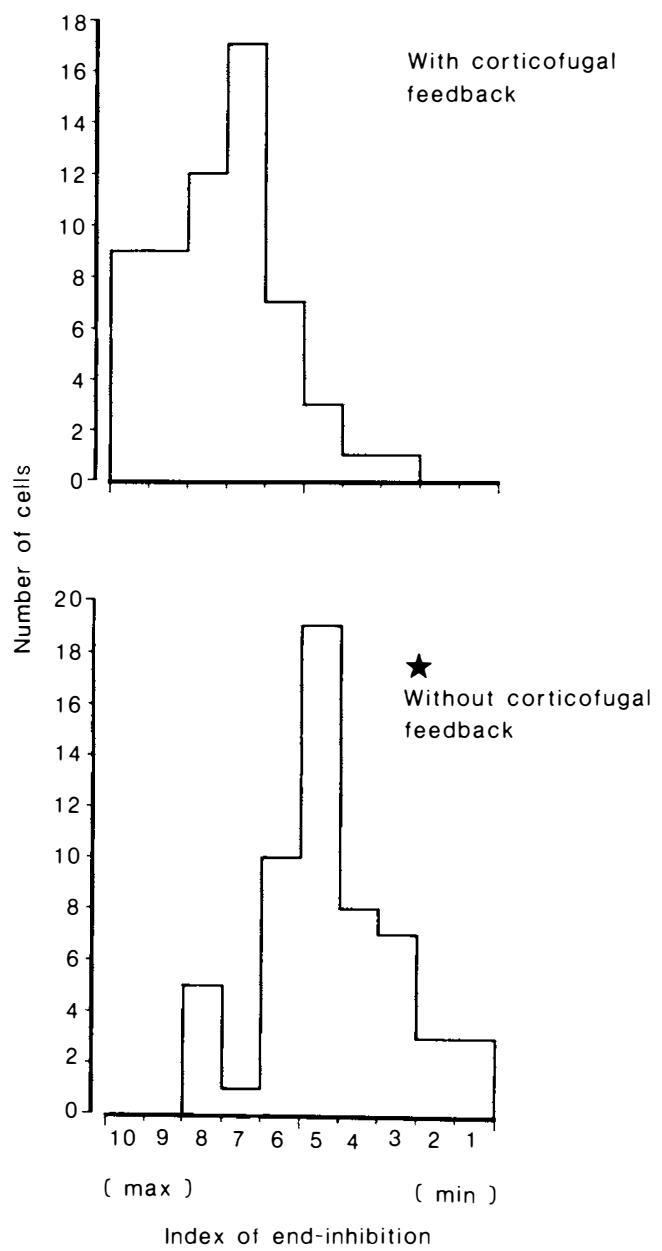

Fig. 4. Histograms summarising the degree of length tuning seen in dLGN cells with and without corticofugal feedback. Length tuning was compared by producing an index of end-inhibition based on the reduction in peak response magnitudes seen at the plateau averaged over $2^{\circ}, 4^{\circ}$ and $6^{\circ}$, (peak-plateau) $x 100 /$ peak. Cells are subdivided into 10 categories of varying end-inhibition: category 1 represents cells with little or no endinhibition, category 10 those showing almost total suppression of the response when bar lengths exceed $2^{\circ}$. Cells in category 6 and above would be considered hypercomplex in the cortex. The population of cells with corticofugal feedback show a mean response reduction of $71 \%(+/-2 \% \mathrm{sem}, N=59)$ and those without $43 \%$ $(+/-2 \%$ s.e. $m ., N=56)$. Note these cells were studied in the same preparations by making simultaneous recordings from left and right $d L G N s$, one with corticofugal feedback and one without. 


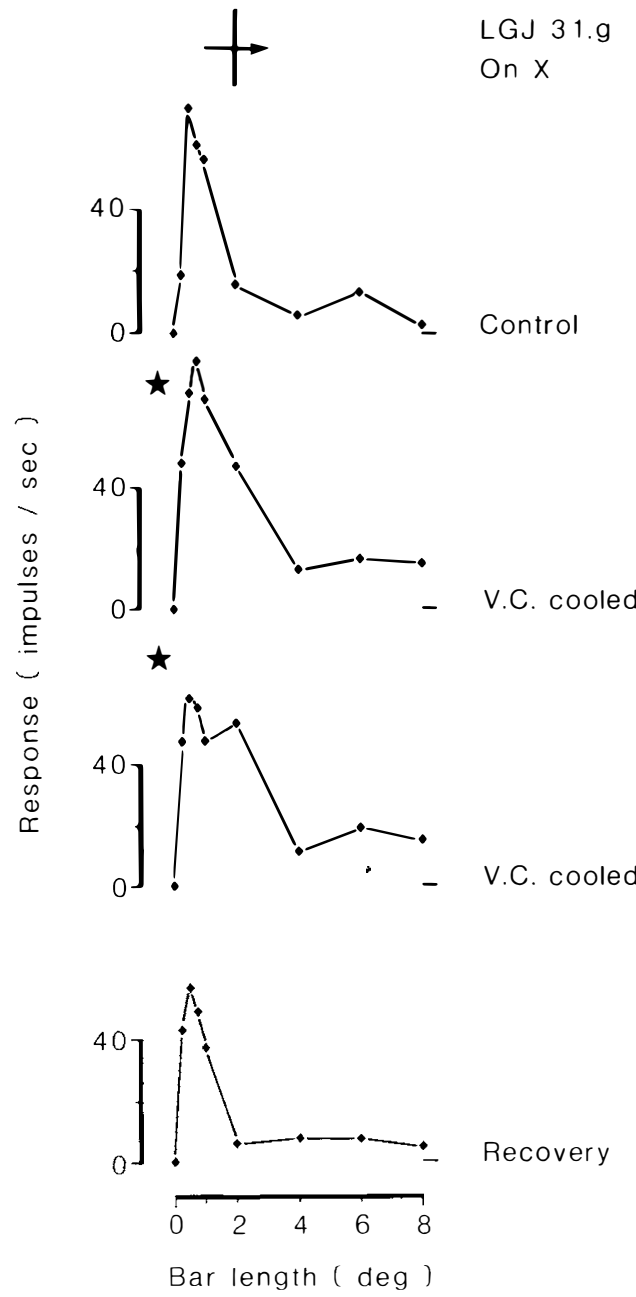

Fig. 5. Effect of partially cooling areas 17 and 18 on the length tuning curve of an on-centre $X$ cell in the $d L G N$. This shows a reversible reduction in length tuning.

visual cortex that cells with over $50 \%$ response reduction should be classified as hypercomplex; cells in categories 6 and above showed a comparable degree of length tuning. The shift between the populations with and without feedback is obvious. The effects shown by the removal of the visual cortex are obviously irreversible. We are also in the process of examining the effects elicited by reversible cortical cooling. It has proved difficult to cool the cortex to the point where activity in layer VI in areas 17 and 18 is completely blocked without darnaging the superficial layers. However we have been able to produce partial blockades of cortical activity that are reversible and an example of the results obtained from one of these is shown in Figure 5. During cortical cooling there was a notable reduction in the tightness of the length tuning curve of this on-centre $\mathrm{X}$ cell and a return to control values after cessation of cooling.

The primary point from the results discussed above is that the corticofugal feedback substantially enhances but does not generate the length tuning of dLGN cells. It is clear that a component can be related to the influence of the centre-surround antagonism seen in the fields of both retinal ganglion and dLGN cells. As a bar of light sweeps over the receptive field it will engage surround as well as centre mechanisms. The surround antagonism will reduce the centre response, and because GABA mediated inhibitory interactions enhance the centresurround antagonism in the $\mathrm{dLGN},{ }^{18}$ this effect will be more pronounced than that occurring at retinal levels. However, a simple consideration of the geometric relationship suggests that for bars of a similar width to the centre mechanism, only a proportion of the surround antagonism will be brought to bear on the centre response. The length tuning generated by this mechanism might therefore be expected to be weak. However corticofugal cells are well activated by such stimuli and a corticofugal drive to the inhibitory input impinging on such a cell could greatly strengthen the inhibitory force brought in from the surround. This suggestion is summarised in somewhat idealised form in Figure 6 . The widespread occurrence of centre-surround mechanisms in all kinds of sensory input supports the view that the selectivity conferred by a process differentially distributed so as to favour the centre of a response field (where the field could represent any variable that is translated into a "topographical" distribution across a population of neurones), is important to higher processing levels. In a very simple spatial sense, dLGN and retinal ganglion cell fields are highly selective to the location and size of a stationary flashing spot. In the absence of corticofugal feedback they are less so to a moving bar, because it brings in less of the surround mechanism. It would appear that corticofugal feedback redresses the balance, increasing selectivity so that responses are 


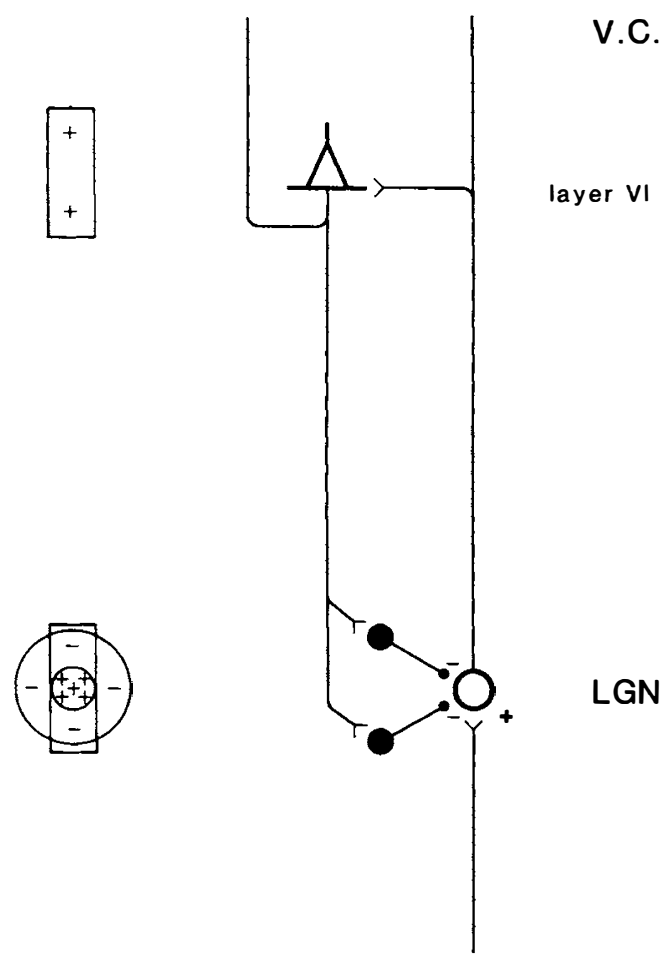

Fig. 6. Diagram to illustrate the possible mechanism of the corticofugal contribution to length tuning in the $d L G N$. To the right is shown a dLGN relay cell receiving excitatory input from a retinal afferent and in turn contacting a layer VI pyramidal cell in the visual cortex. The dLGN cell has a centre-surround type receptive field, as indicated to the left of the figure, while the cortical cell responds preferentially to elongated bars. The dLGN cells also receives an input from intrinsic inhibitory interneurones, which serve to enhance the surround suppression of centre response. Hence a flashing spot covering the entire receptive field will generate inhibition from the surround that almost cancels out the excitation from the centre (quantified here by the distribution of + and - signs in the centre and surround). A bar will cover less of the receptive field surround and so fail to engage the inhibitory mechanisms at retinal and geniculate level as effectively. However, it will also stimulate the cortical cell, generating an excitatory feedback to the $d L G N$ which is presumed to enhance the effectiveness of the inhibitory input generated by the relay cell surround. Hence the cell will show similar degrees of response attenuation for bars and spots that extend beyond the receptive field centre.

strongly biased towards a bar of optimal length moving through the spatial domain of the receptive field centre.
Excitatory amino acid receptors and retinal transmission

Despite early reservations (eg Tebecis), ${ }^{19}$ it is now clear that transmission from retinal ganglion cells to relay cells in the dLGN involves excitatory amino acid receptors. Present thought implicates three distinct receptor systems in the action of excitatory amino acids in the nervous system. They are defined by their selective agonists, and referred to as the N-methyl-Daspartate (NMDA), quisqualate and kainate receptors. ${ }^{20} \mathrm{~A}$ possible fourth class, seen in the retina, is identified by the action of the antagonist L-2-amino-4-phosphonobutyrate (LAP4). The first evidence regarding the receptor mediating the retinal input to the lateral geniculate came from an iontophoretic study in the feline dLGN. ${ }^{21}$ An NMDA receptor antagonist was found to selectively block the visual response, providing strong support for the view that the NMDA receptor mediated the optic nerve input. This work excluded the kainate receptor, but did not provide evidence which could be regarded as decisive regarding a possible role for the quisqualate receptor. The issue of a role for a non-NMDA receptor was recently raised by an investigation carried out on the in vitro rat dLGN slice. It was found that the selective NMDA antagonist, D-2-aminophosphonovalerate (APV), had no effect on the epsp evoked by electrical stimulation of the optic tract, ${ }^{22}$ whilst the non-NMDA excitatory amino acid antagonist -D-glutamylglycine did. Although there may be a species difference, this does query the role of the NMDA receptor.

In order to clarify the situation further we have re-examined this question in the cat $\mathrm{dLGN}$ in vivo, using iontophoretic application of the selective NMDA antagonists APV and 3- $((+/-$ )-2-carboxypiperazin-4-yl)-propyl-1-phosphonic acid (CPP), and the putative quisqualate biased non-NMDA antagonist 6-cyano-7-nitorquinoxaline-2,3-dione (CNQX). The primary experimental findings are clear; the NMDA antagonists can virtually eliminate visual driving in dLGN cells at dose levels that leave the action of iontophoretically applied quisqualate and kainate unaffected, while blocking the response to NMDA. ${ }^{23,24}$ An example of this is given in Figure 7. The records show the response of an on-centre $\mathrm{X}$ cell to a spot of light flashed within the receptive field centre, and to iontophoretic 


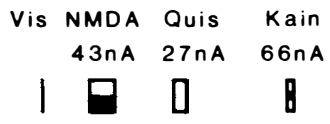

LGC37.d Off $Y$

3 trials
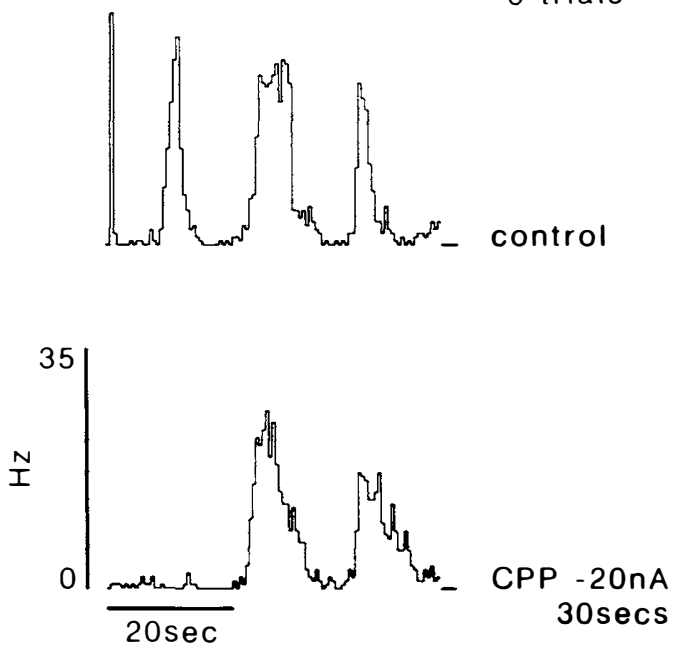

$600 \mathrm{msec}$ bins

Fig. 7. Peristimulus time histograms showing the response of an off-centre $Y$ cell before and during continuous administration of the NMDA antagonist APV, to visual stimulation of the receptive centre and to pulses of NMDA, quisqualate and kainate, averaged over three trials. Drugs were applied iontophoretically, at the currents and durations indicated. Note that the responses to visual and NMDA stimulation were eliminated, without reducing the effectiveness of quisqualate of kainate.

pulses of the agonists NMDA, kainate and quisqualate. The stimulus contrast and drug doses were adjusted to produce more or less equipotent responses under control conditions. During iontophoretic application of the antagonist, CPP, there was a selective loss of the responses to the visual stimulus and NMDA. For a total population of 48 cells studied, we observed a selective block of the action of NMDA and visual reponses in 41 (APV 16/21, CPP 23/27). The remaining cells were also selectively affected in some trials, but showed non-selective effects in others. We observed no difference between X and Y cells or "on" and "off" centre cells. These data clearly underwrite the fact that transmission from retinal afferents to dLGN cells in the cat is critically dependent on the NMDA receptor.
The suggestion that the NMDA receptor plays such a crucial role in the transfer of information in the dLGN has a substantive impact on the way we view the role of the dLGN. In the presence of normal physiological concentrations of magnesium ions the NMDA receptor exhibits a marked voltage dependency. This is because $\mathrm{Mg}^{2+}$ ions exert a pronounced blocking action at potentials near the resting membrane potential, which is alleviated with progressive depolarisation of the cell. ${ }^{25,26}$ Thus the level of membrane deplorisation gates the effectiveness of the NMDA receptor, with the consequence that the transmission of information at the retino-geniculate synapses will be critically dependent on those factors, both visual and nonvisual, that influence the level of dLGN cell depolarisation.

Is the NMDA receptor the only excitatory amino acid receptor involved in the transfer of visual information in the feline dLGN? Previous work in the ventrobasal thalamus has indicated that although NMDA receptors play a major role in the normal response to sensory stimulation, non-NMDA receptors are also involved. It seems that a short latency component of the response, demonstrated best by electrical stimulation of the sensory input or by a very brief sensory stimulus, is resistant to NMDA receptor blockade but sensitive to the broad spectrum excitatory amino acid antagonists. ${ }^{27,28}$ This suggests that the response might be initiated by non-NMDA receptors and then carried by NMDA receptors once the level of depolarisation crosses the threshold necessary to start to lift the magnesium dependent blockade of the channel. It is plausible that this might also apply to the retinal input to the dLGN. If this were the case, however, the short latency components of the visual response should be resistant to the effects of selective NMDA receptor blockade. In most, but not all, cases careful examination of the visual responses on an expanded time scale revealed that one or more spikes in the response were indeed often resistant to NMDA receptor blockade. In certain instances, such as that shown in Figure 8, this involved a very short latency spike occurring within the time domain of the first onset transient. This would be consistent with the results obtained in the ventrobasal thalamus. However this was not so in all instances, and in 


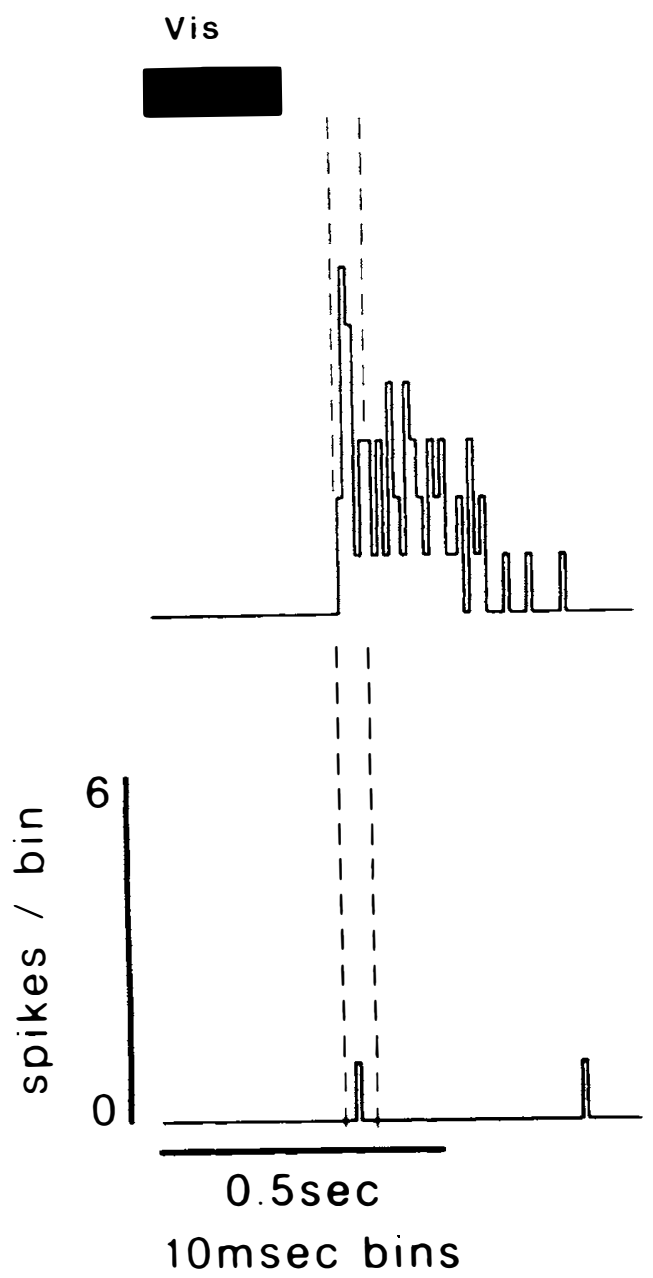

Fig. 8. Visual response of an off-centre $Y$ cell to a spot of light flashed in the receptive field centre under control conditions (upper record) and during selective NMDA receptor blockade (lower record). Vertical interrupted lines define onset transient of the response. Note that although the bulk of the response is lost during blockade of the NMDA receptor, one spike of the onset transient remains. Records show response to one trial.

any case the clearly identified non-NMDA component, where present, was small in comparison to that seen in the vetrobasal thalamus. Use of the "non-NMDA" excitatory amino acid antagonist CNQX provided another means of accessing this issue. We examined the effect of CNQX and found it to be less selective than previous reports suggested.$^{29}$ Nevertheless, we were able to obtain a very significant reduction in visual response magnitude with a dose of the antagonist that substantially reduced the response to quisqualate and kainate, but exerted only a relatively small effect on that to NMDA. ${ }^{24}$ Using an NMDA selective antagonist such a small reduction in the NMDA response would not have been associated with a significant change in visual response. This suggests that the non-NMDA receptors also make a very significant contribution at this synapse. In short, it seems that if either NMDA or non-NMDA receptors are blocked the transmission of visual information through the dLGN will be radically impaired.

Excitatory amino acid receptors and the "synaptic gate" controlling retinal access to the brain

The likelihood that both NMDA and nonNMDA receptors are involved in the visual response, together with the known voltage dependency of the NMDA receptor, seems to present a complex and puzzling situation. However, there does seem to be a pattern emerging from the many facets of information that we can now bring together. The non-NMDA receptors can be regarded as establishing a level of background depolarisation which enables the operation of channels associated with the NMDA receptor. In the in vitro dLGN brain slice (eg Crunelli et al), ${ }^{22}$ there is no background activity in the optic nerve, no corticofugal influence and no brain stem modulatory inputs. Electrical stimulation of the optic tract will provoke a single synchronous volley of input to the dLGN which can be entirely expected to occur via the operation of the non-voltage dependent quisqualate or kainate receptors. In vivo the situation is quite different. Firstly, in the absence of specific patterned visual stimulation, there is constant background barrage of input from the retinal ganglion cells with a mean level in the region of 40 impulses/second. ${ }^{30}$ This means that there will be already a substantive depolarising influence moving the dLGN cells into the range of NMDA receptor operation. Indeed the response to a visual stimulus can be regarded at least in part as modulating this background activity up or down, 
rather than generating a de novo input. Thus for example, a spot of light flashed over a restricted location in visual space will cause respectively a decrement and an increment in the activity of "off" and "on" centre cells with receptive fields in that area. Similar comments would apply to the pattern of effect initiated by a bar of light moved over that location. The visually driven retinal input will reflect the temporal properties of the stimulus "smeared" by the retinal neural network, with attendant "background" activity levels, and can be seen to contrast sharply with the tightly time locked unitary epsp evoked by electrical stimulation of the optic tract in vitro. The in vivo situation is further complicated by the pattern of corticofugal activity and the complex modulatory influence of the brain stem non-specific inputs. One of these, the cholinergic input exerts a direct excitatory effect on dLGN relay cells and an inhibitory influence on the inhibitory interneurones. ${ }^{31,32}$ In its most limited sense of operation, the cholinergic influence clearly carries the potential to move dLGN relay cells into the level of depolarisation compatible with lifting the NMDA receptor blockade, because it can simultaneously disinhibit and directly depolarise them.

As so far presented, the operation of the retinogeniculate synapse can be regarded as following from a background depolarisation level established by non-NMDA receptors and the cholinergic input, on which the NMDA receptor function serves to provide the additional levels of depolarisation necessary to precipitate the dynamic fluxes in action potential discharge associated with the visual input. From this viewpoint it is apparent that removal of either the NMDA or non-NMDA components of the response will have the capacity largely to eliminate the visual response. Equally a neuromodulatory bias of the depolarisation level will, because of the voltage dependency of the NMDA receptor, exert a stronger influence on the visual response than would be anticipated if it were carried solely by non-NMDA receptors. Visual responses are not however formed around simple static shifts up or downwards in excitatory drives. There is a dynamic and complex temporally and spatially modulated pattern of activity change that includes inhibitory as well as excitatory drives. Considering firstly, events mediated solely at the level of the dLGN, both centre and surround mechanisms include a contribution from intrageniculate GABAergic inhibitory processes $^{18}$ and these can be distinguished both in the temporal and spatial sense. Even a simple stimulus such as a bar of light drifting over the receptive field will bring in a wave of inhibition and excitation, varying according to the precise stimulus parameters. The voltage dependency of the NMDA receptor allows the possibility of a significant non-linearity in the effectivness of the inhibitory inputs, whereby lower than anticipated levels of inhibitory drive could exert a pre-emptive control over the capability of the cell to respond to the visual input. Taking note of the corticofugal influence a further dimension is added to the problem. As discussed in the preceding section, using the bar as a simple example, the corticofugal projection will encompass a spatially structured pattern of excitatory and inhibitory influence. According to the length of the bar the relative power of the excitatory and inhibitory influence will vary and the temporal and spatial dynamics of these, in accord with the local geniculate influences will determine the "window" of depolarisation through which the NMDA receptor can translate the retinal output. The very strength of the anatomically defined corticofugal excitatory contacts to the dLGN cells suggest the capability for a massive influence on the level of dLGN cell depolarisation. As the spontaneous activity levels of the corticofugal cells are low, their influence is only to be seen in terms of their visually driven activity. It seems likely that under many conditions only those stimulus configurations that optimise the corticofugal facilitation to a given dLGN locus will allow the NMDA receptor to transmit the visual input. Otherwise the potent and strong inhibitory forces will hold down the membrane potential to levels below the "window".

We should be aware of the likelihood that complex patterns of visual stimulation as encountered in normal behavioural conditions will produce an equally complex pattern of corticofugal feedback. The precise way in which the excitatory and inhibitory components of the influence will be modulated is not clear, but one might anticipate from the available evidence ${ }^{13,14,17}$ that patterned stimulation of a substantial portion of the visual field would 
provoke a powerful inhibitory drive. It is also relevant in this context to consider binocular interactions in the dLGN. Firstly, the corticofugal system itself should provide a strong binocular drive because of the binocular fields of many layer VI. cells (eg Gilbert) ${ }^{11}$ and the fact that individual corticofugal fibres are reported to make synaptic contacts in geniculate layers representating both eyes. ${ }^{33}$ In line with this there is good evidence for powerful binocular inhibitory influences in the dLGN as well as some weak facilitatory effects ${ }^{34,35,36,37}$ but not all these can be attributed to the corticofugal system. ${ }^{38}$ Overall the system seems to be biased towards a level of inhibitory drive that might be expected to pre-empt the effective influence of the NMDA receptor. The cholinergic drive can in this sense be seen to be critical because it affects the set point in the focus of stimulus derived inhibitory and excitatory pattern, diminishing the power of the inhibitory drive in a way which may align the relay cell membrane potentials with the operating range of the NMDA receptor whilst permitting the peaks of the inhibitory forces to structure the response.

The support of the MRC and Wellcome Trust is gratefully acknowledged.

\section{References}

${ }^{1}$ Wilson JR, Friedlander, MJ, Sherman SM. Fine structural morphology of identified X- and Y-cells in the cat's lateral geniculate nucleus. Proc Roy Soc Lond B 1984; 221: 411-36.

${ }^{2}$ Famiglietti EV and Peters A. The synaptic glomerulus and the intrinsic neurons in the dorsal lateral geniculate nucleus of the cat. J Comp Neurol 1972; 144: 285-322.

${ }^{3}$ Rapisardi SC and Miles TP. Synaptology of retinal terminals in the dorsal lateral geniculate nucleus of the cat. J Comp Neurol 1984; 223: 515-34.

${ }^{4}$ Ahlsen G, Grant K, Lindstrom S. Monosynaptic excitation of principal cells in the lateral geniculate nucleus by corticofugal fibres. Brain Res $1982 ; 234$ : 454-8.

${ }^{5}$ De Lima AD and Singer W. The serotinergic fibres in the dorsal lateral geniculate nucleus of the cat: Distribution and synaptic connections demonstrated with immunocytochemistry. J Comp Neurol 1987; 258: 339-51.

${ }^{6}$ De Lima AD and Singer W. The brain stem projection to the lateral geniculate nucleus in the cat: Identification of cholinergic and monoaminergic elements. J Comp Neurol; 1987; 259: 92-121.

${ }^{7}$ Kromer LF and Moore RY. A study of the organisation of the locus coeruleus projections to the lateral geniculate nuclei in the albino rat. Neuroscience 1980; 5: 255-71.

${ }^{8}$ Kalil RE and Chase R. Corticofugal influence on activity of lateral geniculate neurons in the cat. $J$ Neurophysiol 1970; 33: 459-74.

${ }^{9}$ Geisart EE, Langsetmo A, Spear PD. Influence of the cortico-geniculate pathway on response properties of cat lateral geniculate neurons. Brain Res 1981; 208: 409-15.

${ }^{10}$ Hubel DH and Wiesel TN. Receptive fields, binocular interaction and functional architecture in the cat's visual cortex. J Physiol 1962; 160: 106-54.

"Gilbert CD. Laminar differences in receptive field properties of cells in cat primary visual cortex. $J$ Physiol 1977; 268: 391-421.

${ }^{12}$ McClurkin JW and Marrocco RT. Visual cortical input alters spatial tuning in monkey lateral geniculate nucleus cells. $J$ Physiol 1984; 348: 135-52.

${ }^{13}$ Marrocco RT and McClurkin JW. Evidence for spatial structure in the cortical input to monkey lateral geniculate nucleus. Exp Brain Res 1985; 59: 50-6.

${ }^{14}$ Tsumoto T, Creutzfeldt OD, Legendy CR. Functional organisation of the corticofugal system from visual cortex to the lateral geniculate nucleus in the cat. Exp Brain Res 1978; 32: 345-64.

${ }^{15}$ Cleland BG, Lee BB, Vidyasager TR. Response properties of neurones in the cat's lateral geniculate nucleus to moving bars of different length. $J$ Neurosci 1983; 3: 108-16.

${ }^{16}$ Jones HE and Sillito AM. The length tuning of cells in the feline dorsal lateral geniculate nucleus (dLGN). J Physiol 1987; 390: 32P.

${ }^{17}$ Murphy PC and Sillito AM. Corticofugal feedback influences the generation of length tuning in the visual pathway Nature 1987; 329: 727-9.

${ }^{18}$ Sillito AM and Kemp JA. The influence of GABAergic inhibitory processes on the receptive field structure of $\mathrm{X}$ and $\mathrm{Y}$ cells in the cat dorsal lateral geniculate nucleus (dLGN). Brain Res 1983; 277: 63-77.

${ }^{19}$ Tebecis AK. Studies on the identity of the optic nerve transmitter. Brain Res 1973; 63: 31-42.

${ }^{20}$ Watkins JC and Evans RH. Excitatory amino acid transmitters. Ann Rev Pharmacol Toxicol 1981; 21: 165-204.

${ }^{21} \mathrm{Kemp} \mathrm{JA}$ and Sillito AM. The nature of the excitatory transmitter mediating $\mathrm{X}$ and $\mathrm{Y}$ cell inputs to the cat dorsal lateral geniculate nucleus $J$ Physiol 1982; 323: 377-91.

${ }^{22}$ Crunelli V, Kelly JS, Leresche N, Pirchio M. On the excitatory post-synaptic potential evoked by stimulation of the optic tract in the rat lateral geniculate nucleus. $J$ Physiol 1987; 384: 603-18. 
${ }^{23}$ Moody CI and Sillito AM. The role of the Nmethyl-D-aspartate (NMDA) receptor in the transmission of visual information in the feline dorsal lateral geniculate nucleus (dLGN). J Physiol 1987; 396: 62P.

${ }^{24}$ Sillito AM, Murphy PC, Salt TE, Moody CI. The relative dependence of retino-geniculate transmission on the NMDA receptor. (In preparation.)

${ }^{25}$ Nowak L, Bregestovski P, Ascher P, Herbert A, Prochiantz A. Magnesium gates glutamate activated channels in mouse central neurons. Nature 1984; 307: 462-5.

${ }^{26}$ Mayer ML and Westbrook GL. The action of Nmethyl-D-aspartate on mouse spinal neurones in culture. J Physiol 1985; 361: 65-90.

${ }^{27}$ Salt TE. Mediation of thalamic sensory input by both NMDA receptors and non-NMDA receptors. Nature 1986; 322: 263-5.

${ }^{28}$ Salt TE. Excitatory amino-acid receptors and synaptic transmission in the rat ventrobasal thalamus $J$ Physiol 1987; 391: 499-510.

${ }^{29}$ Honore T, Davies SN, Drejer J, Fletcher EJ, Jacobsen P, Lodge D, Nielson FE. Potent and competitive antagonism at non-NMDA receptors by FG 9041 and FG 9065. Soc Neurosci Abstr 1987; p 383.

${ }^{30}$ Kaplan E, Purpura K, Shapley RM. Contrast affects the transmission of visual information through the mammalian lateral geniculate nucleus. $J$ Physiol 1987; 391: 267-88.
${ }^{31}$ Sillito AM, Kemp JA, Berardi N. The cholinergic influence on the function of the cat dorsolateral geniculate nucleus (dLGN). Brain Res 1983; 280 : 299-307.

${ }^{32}$ McCormick DA and Pape H-C. Acetylcholine inhibits identified inhibitory interneurones in the cat lateral geniculate nucleus. Nature. (In Press.)

${ }^{33}$ Robson, JA. The morphology of corticofugal axons to the dorsal lateral geniculate nucleus in the cat. J Comp Neurol 1983; 216: 89-103.

${ }^{34}$ Sandersen KJ, Bishop PO Darian-Smith I. The properties of the binocular receptive fields of lateral geniculate neurons. Exp Brain Res 1971; 13: 178-207.

${ }^{35}$ Schmielau F and Singer W. The role of visual cortex for binocular intractions in the cat lateral geniculate nucleus. Brain Res 1977; 120: 354-61.

36 Burges J, Grieve KL, Murphy PC, Sillito AM. Iontophoretically applied bicuculline reveals excitatory responses from the non-dominant eye receptive fields of cells in the A laminae of the cat dorsal lateral geniculate nucleus (dLGN). $J$ Physiol 1985; 369: 36P.

${ }^{37}$ Eysel UT, Pape H-C Van Schayk R. Excitatory and differential disinhibitory actions of acetylcholine in the lateral geniculate nucleus of the cat. $J$ Physiol 1986; 370: 233-54.

${ }^{38}$ Murphy PC and Sillito AM. The source of the input generating binocular excitation in the $\mathrm{A}$ laminae of the feline dorsal lateral geniculate nucleus (dLGN). J Physiol 1987; 390: 35P. 